\title{
SATURATION AND ADAPTATION IN THE ROD SYSTEM
}

\author{
Edward H. Adelson* \\ Vision Research Laboratory, University of Michigan, Department of Psychology, Ann Arbor, MI 48109, U.S.A.
}

(Received 16 July 1981; in revised form 5 March 1982)

\begin{abstract}
A background that is briefly flashed to a dark-adapted eye saturates the rod system. This transient saturation occurs with backgrounds that are as much as $2 \log$ units dimmer than those producing saturation under steady viewing. Rod threshold is highest when the background is first turned on, and falls as adaptation proceeds. The nature of the adaptive processes are studied by presenting flashed backgrounds on pre-adapting fields. The data can be interpreted in terms of two adaptive processes: the first is multiplicative, and occurs rapidly; the second is subtractive, and occurs more slowly.
\end{abstract}

\section{INTRODUCTION}

The retina is required to function under light levels that span an enormous range, and to do this effectively it must adapt to whatever ambient light level is present. In the cone system an important source of adaptation at high light levels is photopigment bleaching: as the pigment is depleted, the cones catch fewer quanta, and so give smaller responses. Indeed, pigment depletion seems to prevent cone signals from saturating under steady adaptation, as was shown by Alpern et al. (1970b). Those investigators also pointed out that, since the bleaching process takes time to come to equilibrium, one might expect that cone saturation would occur if thresholds were measured on a background that was flashed, rather than being steadily present. This effect had actually been observed by Brindley, who hinted at it in Brindley (1959), without showing the relevant data points, and then showed it clearly in Brindley (1963), where he replotted some of his 1959 data. Brindley found cone saturation to occur with flash intensities of about $200 \mathrm{~cd} / \mathrm{m}^{2}$, with a $4 \mathrm{~mm}$ pupil, which converts to about $3.4 \log$ td-sec. This value is similar to that later measured by Shevell (1977). (See also King-Smith and Webb, 1974; Geisler, 1978.)

Hood and his co-workers (Hood, 1978; Hood et al., 1978), and Geisler (1978a, 1980) have found evidence that other adaptive mechanisms, besides pigment depletion, can also help prevent cone saturation. They have related these findings to the large body of electrophysiological evidence on adaptation in photoreceptors (e.g. Kleinschmidt and Dowling, 1975; Norman and Werblin, 1974).

Earlier psychophysical workers have also investigated the initial effects of a background flash, and

*Present address: RCA, David Sarnoff Research Center, Princeton, NJ 08540, U.S.A. have traced out the time-course of the effects of light adaptation. Crawford (1947) measured foveal increment thresholds against a flashed field, and found a large transient threshold elevation during the first $100 \mathrm{msec}$ of background presentation (indeed, the flashed background paradigm employed in the present study is sometimes known as "Crawford masking"). Boynton and Kandel (1957) measured thresholds with flashed backgrounds, and found that if the eye was adapted to a "pre-adapting" level before the flash appeared, threshold elevation could be reduced (but see Sperling, 1965). Although Boynton and Kandel did not reach saturating conditions, the adaptive effects they observed are no doubt related to those that Hood and Geisler found would reduce the tendency to saturation in cones.

The present study is concerned with rods, rather than cones, and so the situation is somewhat different. The rod system does show saturation in steady state (Aguilar and Stiles, 1954) and so one must conclude that whatever adaptive mechanisms are present in the rod pathway are insufficient to prevent the signals from saturating. Photopigment depletion will not affect rod sensitivity via reduced quantum catch, since only insignificant amounts of pigment are bleached below saturation. There is some evidence that vertebrate rods show no adaptation at all (Dowling, 1967; Penn and Hagins, 1972), although the evidence on this is uncertain (see appendix II), and in any case leaves open the question of adaptation beyond the receptors.

One way of studying adaptation in the rod system is to ask whether rod saturation is any different when the backgrounds are flashed, rather than steady. As was first reported in a preliminary description of the present work (Adelson, 1977). the rod system can saturate with flashed backgrounds that are some 100 times dimmer than those that cause saturation in stcady state. In the experiments that will be described, 
rod saturation was studied at various times after the onset of a background, in an attempt to understand some of the adaptive processes that occur between the onset of the background and the ultimate state of adaptational equilibrium.

\section{METHODS}

All experiments were done on the Maxwellian view system shown in Fig. 1. The system normally had 2 channcls, but a third channcl could be added by the insertion of the beam splitter BS', shown in dashed lines.

A tungsten ribbon filament, $\mathrm{I}$, was run from a constant voltage power supply. Lens $L$ imaged the filament onto pinhole $\mathrm{P}$, which actually consisted of two pinholes, one above the other, each selecting one portion of the elongated ribbon image. The beam from the top pinhole continued in a straight line, and encountered shutter $S_{1}$ (Uniblitz; Vincent Associates). aperture $A_{1}$, and neutral density wedge $W_{1}$ (Inconel; Bausch \& Lomb). Mirror $\mathrm{M}_{1}$ introduced a $90^{\circ}$ turn, and the beam proceeded through the filters in filter holder $\mathrm{FH}_{1}$, the optional beam splitter BS (if used), the combining beam splitter BS, and then to the observer's eye (O) through filter holder $\mathrm{FH}_{4}$ and Maxwellian lens ML. ML imaged the pinhole $P$ in the plane of the eye's entrance pupil, and imaged the field stop FS at optical infinity.
The second beam followed a similar path, except that it emerged from the lower pinhole at $P$, and was immediately reflected at $90^{\circ}$ by mirror $M_{0}$; it then continued its route through $\mathrm{S}_{2}, \mathrm{~A}_{2}, \mathrm{~W}_{2}, \mathrm{M}_{2}, \mathrm{FH}_{2}, \mathrm{FS}$ (the field stop, defining the test patch), BS, $\mathrm{FH}_{4}$ and ML.

When a third channel was needed, the optional beam splitter BS $^{\prime}$ was inserted, and the extra tungsten lamp $T^{\prime}$ was turned on. This lamp (Mazda F-58) had its filament arranged in a compact square, and the flament itself was imaged in the plane of the subject's entrance pupil (without the lens-pinhole system that was used with the other channels).

The observer stabilized his head by biting on a bite bar, which had an impression of his teeth in dental compound. With his right eye, he fixed on a fixation point (FP) which was generated by a tiny light emitting diode, imaged at optical infinity by a small lens. and encased in a metal tube.

For most of the experiments, the author was the subject; one other subject was sometimes used. Both subjects were well-corrected myopes, and wore their spectacles during the experiments.

\section{Calibration of filters and light levels}

Four types of filters were used: neutral density inconels (Bausch \& Lomb); neutral density Wratten No. 96 gelatins (Eastman Kodak, Rochester, NY); colored gelatines (Eastman Kodak and Ilford Ltd.,

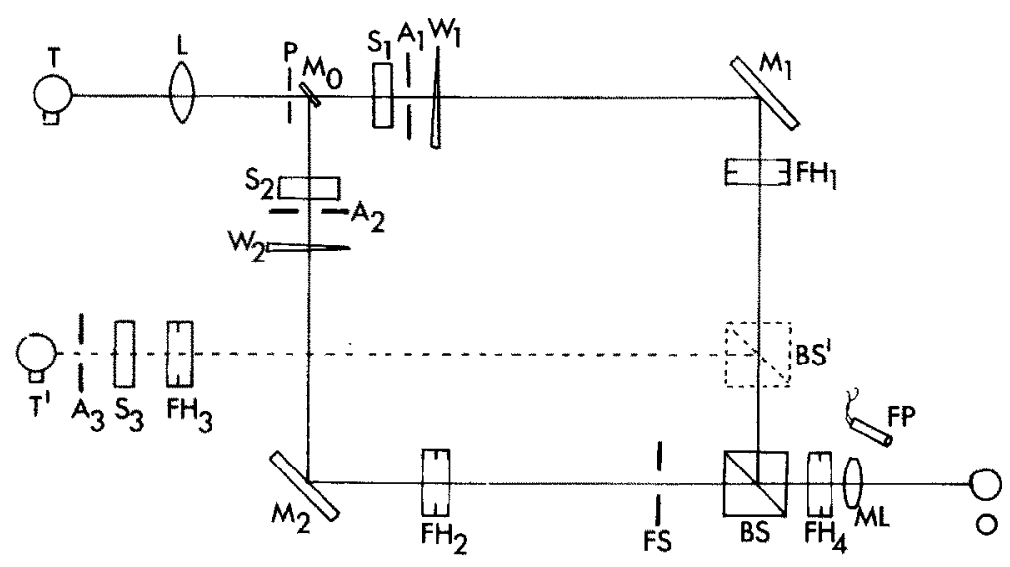

a.

b.

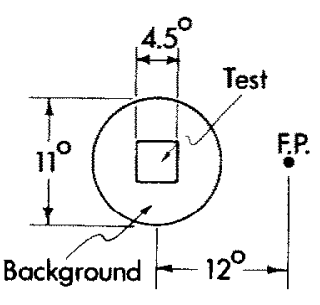

Fig. 1. (a) The Maxwellian view system. See text for details. (b) The observer's view in the apparatus. The test patch was $4.5^{\circ}$ square, and was centered on the $11^{\circ}$ background field. The fixation point (F.P.) was $12^{\circ}$ to the right of the ficld. 
Essex, U.K.); narrow band interference filters (Baird Atomic). The spectral transmittances of all filters were calibrated using a Beckman Acta II spectrophotometer.

The scotopic densities of the colored gelatins were measured in the apparatus by the method of Flamant and Stiles (1948). The scotopic effectiveness of the filtered light was compared with that of white light, by determining the amount of light needed to raise the scotopic threshold by $1.0 \mathrm{log}$ unit. The relative scotopic densities of the interference filters were measured in the apparatus with a PIN-10 photodiode, using the quantized CIE scotopic luminosity function. The absolute scotopic densities of two interference filters were then measured by the method of Flamant and Stiles, and all the other interference filter densities were normalized against these.

Absolute light levels, in scotopic trolands, were measured in two ways. First, the absolute calibration of the PIN-10, supplied by the manufacturer, was used to derive quanta $\sec ^{-1} \mathrm{deg}^{-1}$ with a given interference filter in place. This was converted to scotopic trolands (Wyszecki and Stiles, 1967) and the scotopic density of the filter was added to this value to give the scotopic retinal illuminance with the unfiltered white light. The second technique was photometric, as described by Westheimer (1966). The unfiltered light was projected onto a white plate of known reflectance, and its luminance was measured with a Macbeth Illuminometer, which had been calibrated against a standard source. This value was then converted to scotopic trolands, after a correction for color temperature (Wyszecki and Stiles, 1967). These two methods gave slightly different values, disagreeing by $0.2 \mathrm{log}$ units. The mean value was used.

Daily calibration checks were made with an SEI photometer that was calibrated against the Macbeth Illuminometer. Day to day variability was very small, usually less than $0.05 \log$ unit, indicating that the combination of tungsten filament and constant voltage supply gave a very stable light source.

\section{General procedure}

Unless otherwise noted, the procedure in the experiments was as follows.

The subject's pupil was dilated with Mydriacyl (Alcon Labs, Fort Worth, TX) or Cyclogel (Alcon Labs). He dark-adapted for 30 min prior to the start of the experiment.

After biting on the bite bar, the subject positioned his head so that the adapting field entered through the center of his pupil. The center was found by moving the positioning vise that held the bite bar, in the $X$ and $Y$ directions, and finding the points at which the pupil began to occlude the beam on either side. The midway point was then computed from the readings taken at the index marks on the vise controls. The test beam was positioned to enter the pupil $3 \mathrm{~mm}$ to the nasal side of center, using the mirror $\mathrm{M}_{2}$.
The subject triggered all trials with a hand-operated switch. He could control wedge $W_{2}$ with a servo motor, and wedge $\mathrm{W}_{1}$ with a mechanical linkage.

In all the experiments to follow, an attempt was made to isolate the rod system, and to prevent the cones from detecting the test flash. The conditions adopted were similar to those of Aguilar and Stiles (1954).

As shown in Fig. 1b, the background subtended $11^{\circ}$ and appeared in the nasal half-field of the retina, centered $12^{\circ}$ from the point of fixation. This background was red (Wratten No. 26 gelatin; dom. wave. $621 \mathrm{~nm}$ ). To adapt the cones maximally, the background light entered the subject's eye through the center of the pupil. The background could either be steadily present (as in Aguilar and Stiles' experiment), or flashed.

The test was a square, $4.5^{\circ}$ on a side, and fell in the center of the background. To excite the rods preferentially, rather than the cones, it entered through the nasal edge of the subject's pupil. The test duration was always $30 \mathrm{msec}$.

The choice of test wavelength is important in rod isolation. In the case of steady backgrounds, one must fit the saturating rod branch into a crook formed between the "green" $\left(\Pi_{4}\right)$ and "blue" $\left(\Pi_{3}\right)$ cone mechanisms, and Aguilar and Stiles found that a wavelength of about $520 \mathrm{~nm}$ did this best; accordingly, an Ilford No. 624 gelatin filter (dom. wave. $524 \mathrm{~nm}$ ) was used in this condition. On the other hand, with the transient backgrounds, saturation occurs at a much lower background level, and one tends to run into the "green" cones before the "blue" ones. Therefore, it is advantageous to use a shorter wavelength test flash; a $480 \mathrm{~nm}$ test was found to give good rod isolation in the transient conditions.

\section{EXPERIMENT 1. TRANSIENT ROD SATURATION}

The first task is to measure the point at which rod saturation occurs when the background is flashed, and to compare it with the case where the background is steadily present. The steady condition is essentially a replication of Aguilar and Stiles (1954) experiment, with slight modifications to fit the present circumstances. In the transient case, the background and test were flashed simultaneously to a dark adapted eye, so that the retina had no opportunity to adapt to the background.

\section{Procedure}

The background was red (Wratten No. 26); the test was green (Ilford No. 624) in the steady condition, and was blue $(480 \mathrm{~nm})$ in the transient condition. The test was $30 \mathrm{msec}$ in both conditions. When the background was flashed, its onset was simultaneous with the test onset, and it remained on for $400 \mathrm{msec}$ to prevent discrimination in the positive rod afterimage. 
Thresholds were taken by the method of adjustment. In the steady condition, the subject adapted to the background for $2 \mathrm{~min}$ before making the threshold judgments. In the flashed background condition, the subject remained in the dark between presentations, in order to remain dark adapted; he also waited for all traces of afterimages to disappear before initiating the next presentation. From time to time. the subject would wait in the dark much longer than usual, to verify that thresholds were not disturbed by residual light adaptation.

The subject triggered each trial when he was ready, and decided whether or not the test flash was discriminable. For a given intensity of flashed background, the subject would adjust the intensity of the test flash over successive trails, until he found the intensity for which it was just discriminable when presented against the background flash.

One unexpected problem arose: in the flashed condition, near saturation, the test would sometimes appear as a darker patch rather than a lighter one. This darkening would typically become visible after $50-100 \mathrm{msec}$ into the background flash. Since it seemed to represent a rather different phenomenon than the usual flash detection, subjects were instructed to ignore it, and to require that the test appear lighter than the background for threshold. (This very interesting subjective phenomenon will be taken up later in the discussion). Subjects were also told to ignore afterimages, and to make judgments only in the initial image.

\section{Results}

Figure 2 shows the results for one subject, averaged over two runs. The steady background condition (solid circles) showed the classical rod saturation

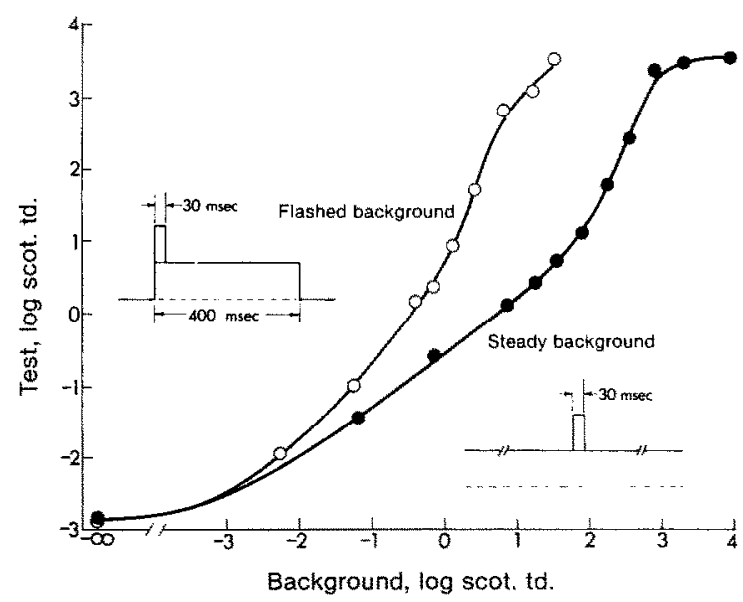

Fig. 2. Increment thresholds, using rod isolation conditions, for the flashed and steady backgrounds of Experiment 1 . Open circles: $30 \mathrm{msec} 480 \mathrm{~mm}$ test against red (Wratten No. 26) background flash. Solid circles: $30 \mathrm{msec}$ green (Ilford No. 624) test against red (Wratten No. 26) background. With flashed backgrounds, rod saturation occurred with much less intense backgrounds than were required with the steady backgrounds. Subject E.A. effect: between 2.5 and $3.0 \log$ scot. td, the increment threshold curve began to rise very steeply. At background levels below saturation, the data did not show a slope of 1 , as they did (or nearly did) in the original experiment of Aguilar and Stiles. This is apparently due to the shorter and smaller test used in the present experiments (cf. Barlow, 1958; also Hallett, 1969); Aguilar and Stiles used a test with 12 times the area and 6.5 times the duration of the one used here.

In the transient case (open circles), increment thresholds rose somewhat more stceply than Weber's law (slope about 1.2) and then showed a steep ascent when the background reached $0.5 \log$ scot. td. The extrapolated curve would asymptote somewhere near $1.0 \log$ scot. td. Thus, transient saturation occurred when the background was fully $2 \log$ units dimmer than the background that caused saturation in steady state.

Since the transient saturation effect was first reported (Adelson, 1977), other investigators have described results taken from similar conditions. In some cases, the transient saturation is found to occur at a higher background intensity than reported here (e.g. Geisler, 1979, 1980; Alexander, 1980). The discrepancy may result from the use of different criteria in the method of adjustment. The problem will be taken up later in this paper.

\section{EXPERIMENT 2. ROD-CONE INDEPENDENCE}

One would like to know whether the transient effects are rod specific, and do not involve rod-cone interactions. Therefore, I measured the action spectrum for the background intensity needed to elevate test threshold to $0.5 \log$ scot. td. This is the point just before transient saturation shows itself (it is difficult to do such a test on the saturating branch itself, because the necessary deviations from rod isolation conditions tend to allow cone intrusion, which would invalidate the procedure).

\section{Procedure}

The conditions were the same as the transient conditions of Experiment 1: the background appeared for $400 \mathrm{msec}$, and the $30 \mathrm{msec}$ test appeared simultaneously with the onset of the background flash. The test was always $480 \mathrm{~nm}$, fixed at $0.5 \log$ scot. td. The background wavelength was varied, and the subject adjusted the background intensity on successive trials, to bring the test to threshold.

\section{Results}

The resulting action spectrum is shown in Fig. 3. It fits the CIE scotopic luminosity function fairly well (shown by the solid line). Thus, rods appear to be elevated by rods. and not by cones, at least at this intensity. Since a small increase in background intensity causes saturation, it is likely that rod signals are themselves responsible for transient rod saturation. 


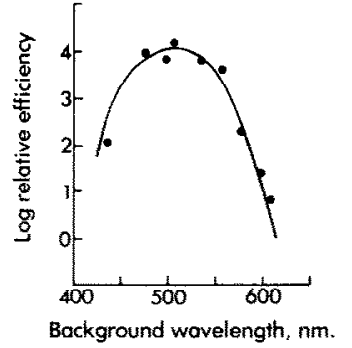

Fig. 3. The intensity of a background flash required to elevate rod threshold to $0.5 \mathrm{log}$ scot. td, as a function of the background wavelength. The solid curve is the quantized CIE luminosity function, which gives a reasonably good fit, indicating that rod signals alone are elevating rod thresholds. Subject E.A.

Rod-cone independence in similar conditions has been reported by Hallett (1969) and White et al. (1978). On the other hand, Alexander (1980) has reported evidence that long-wave cones are important in elevating rod thresholds in transient conditions; in particular, he finds that protanopes show little if any of the transient saturation observed in normal eyes. Rod-cone interactions, if they are involved in the present experiments, will complicate the mapping between physiology and psychophysics, but they will not upset the basic analysis, which is functional rather than physiological.

\section{EXPERIMENT 3. TRANSIENT SATURATION WITH DELAYS}

This experiment extends the results of Experiment 1. As before, test flashes were presented against flashed backgrounds, but now the tests appeared at various times after the onset of the background flash.

\section{Procedure}

The conditions were almost the same as the transient conditions of experiment 1 . A $30 \mathrm{msec}$ test of $480 \mathrm{~nm}$ appeared against a red (Wratten No. 26) background flash. The test onset was delayed by 200 , 500 or $1000 \mathrm{msec}$ after the onset of the background flash. The background always remained on for $400 \mathrm{msec}$ after the test onset. For a given background intensity, the subject adjusted the test intensity on successive trials to bring it to threshold detectability.

\section{Results}

Figure 4 shows the average of two runs on one subject. The data of Experiment 1 have been included in this figure as well; they are the 0 delay and steady state conditions. In addition, the log Weber fractions for each condition are plotted in the lower right.

After $200 \mathrm{msec}$ (open circles), the point of saturation moved rightward by almost a full log unit, indicating a rapid initial recovery from the transient. Between 200 and $1000 \mathrm{msec}$, adaptation continued, and the background intensity for saturation shifted more slowly to the right. But even after $1000 \mathrm{msec}$,

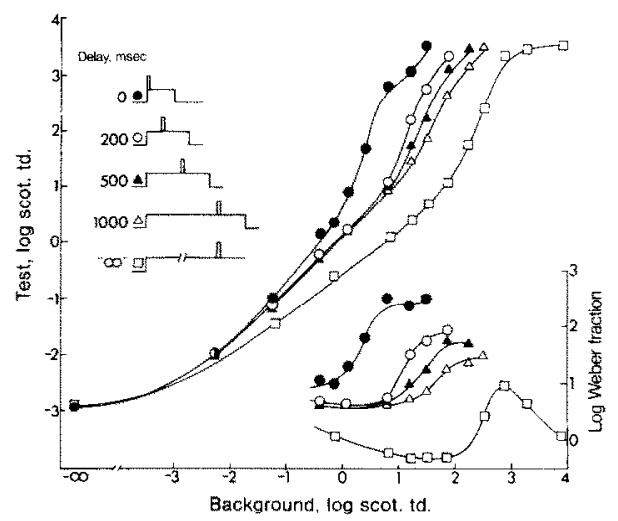

Fig. 4. Increment thresholds measured at various intervals after the onset of the background flash. Successive curves show delays of $0,200,500,1000 \mathrm{msec}$ and "infinity" (i.c. steady state). The corresponding log Weber fractions are plotted in the lower right. Subject E.A.

adaptation was far from complete, and it took 2 or $3 \mathrm{~min}$ for the saturating background intensity to reach its asymptotic value.

\section{Discussion}

Between the time that the background was first turned on, and the time that equilibrium was reached, there must have been large adaptational changes in the rod system. The scheme of Fig. 5 shows, in a qualitative way, how the transient saturation effects could occur.

Suppose that the initial process of visual response is linear with light intensity, but that there is a later stage which imposes a saturating ceiling on the size of the response (cf. Naka and Rushton, 1966; Penn and Hagins, 1972). Further, suppose that there is an adaptational mechanism interposed between the linear stage and the saturating stage, which reduces the size of large signals in order to keep them within the linear range of operation. If this adaptation takes time to turn on, then one can have the situation illustrated in Fig. 5. When a background flash is first turned on
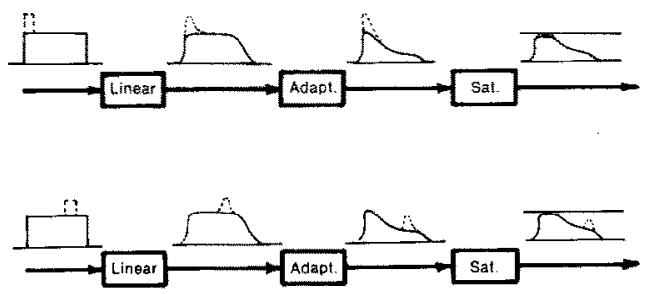

Fig. 5. A qualitative model for transient saturation. The first stage represents a linear transduction process; the second stage is an adaptive process that turns on over time; the third stage is a saturating non-linearity. The curves above the models show the time-course of the light input (extreme left), and the successive transformations of the response at each stage. The solid curve is the background signal; the dotted curve is that of the test. The only difference between the situations shown above and below is that in the latter case the test presentation is delayed, and so the test signal escapes saturation. 
(top), it causes a large response, which will cause saturation if the flash is sufficiently bright. A test increment superimposed upon this initial responsc will be invisible, since the response is already maximal and can go no higher (top, right). However, the adaptive process turns on over time, and reduces the size of the background signal, pulling it back below the ceiling. Thus, if one delays the onset of the test. so that it appears after the adaptation has had time to take effect (bottom), the test can now be detected, since it is able to produce an increment in the size of the nonsaturated output (bottom, right)

One can imagine two mathematically simple processes that could achieve such adaptive effects. The first would be an automatic gain control, which attenuated the signals from both the background and the test, by multiplying them by some fraction less than 1 . The second would be a linear process, which subtracted away part or all of the steady background signal, but allowed transient signals to pass unattenuated (the capacitive coupling found on the "a.c." input of an oscilloscope belongs to this mathematical class, as is shown in appendix 1). The next section formalizes these models and explores some of their predictions.

\section{MODELS FOR SATURATION AND ADAPTATION}

The models can be made more quantitative, using some ideas from electrophysiology. Many retinal cells display a response versus intensity curve of the following form

$$
\frac{V}{V_{\max }}=\frac{I}{I+\sigma}
$$

where $V$ is the response voltage for current in some cases), $I$ is the intensity of the flash of light, and $\sigma$ is the semisaturation constant. Naka and Rushton (1966) originally introduced this equation to deal with $\mathrm{S}$-potentials in fish; it has since been found to dcscribe the responses of cones (Tomita, 1968; Baylor and Fourtes, 1970), rods (Dowling and Ripps, 1972; Penn and Hagins, 1972), and bipolar cells (Werblin and Dowling, 1969). In some cases the data are better fit by an equation of form

$$
\frac{V}{V_{\max }}=\frac{I^{n}}{I^{n}+\sigma^{n}}
$$

where $n$ is an exponent between 0.7 and 1 (Boynton and Whitten, 1970; Norman and Werblin, 1974), but for the present discussion $n$ will be assumed to equal unity.

In studies of photoreceptor adaptation, it has generally been found that a steady background can change the response function in two ways. First of all, the response range may be compressed, which is to say that $V_{\max }$ may be reduced, where $V_{\max }$ is measured as the maximum excursion from the baseline established by the adapting field. Secondly, there may be an increase in the value of $\sigma$, which is to say that it may now take a brighter light to cause a half-saturating response than it did before adaptation. More complex changes may occur as well, but these two are most commonly found (Kleinschmidt and Dowling, 1975; Norman and Werblin, 1974).

Hood (1978) and Hood et al. (1978) have analyzed psychophysical experiments on cone saturation in a framework based on equations (1) and (2), and have found that their data can be understood in terms of shifts in $V_{\text {max }}$ and $\sigma$ (see also Geisler, 1981). A similar analysis will be used here, except that the parameterization will be somewhat different.

Consider a linear system followed by a saturation in the form of equation (1), and let the dark adapted value of $V_{\max }$ be equal to 1 (without loss of generality). Following Hood et al. (1978) (with slight changes in notation), suppose that increment detection consists in detecting the arithmetic difference between the responses from two different regions of the retina, one containing the test plus background, and one containing the background alone, so that the criterion for detection is

$$
V_{1}-V_{b}=\ddot{b}
$$

where $V_{t}$ is the response to the test region (containing both background and test), $V_{b}$ is the response to the background region, and $\delta$ is the threshold for detection.

Then

$$
\frac{I+k I_{t}}{\left(I+k I_{t}+\sigma\right)}-\frac{l}{I+\sigma}=\delta
$$

where $I$ is the background intensity, $I_{t}$ is the added test intensity, and $k$ is a scale factor to take into account the fact that the background and test have different durations and sizes. This leads directly to

$$
I_{t}=\frac{\delta(I+\sigma)^{2}}{k[\sigma-\delta(I+\sigma)]}
$$

(see also Williams and Gale, 1977). This scheme, with its underlying assumptions, may be used to interpret psychophysical data, to determine whether they are compatible with the models that have been used in physiology.

The next question is: how will steady adapting lights change the response functions, and how will these changes affect the corresponding increment threshold curves?

The simplest kind of adaptation is "response compression", illustrated in Fig. 6, which, in a sense, is not adaptation at all. If a steady background is imposed on the system, it induces a steady bias. Since the foor is raised, but the celling is fixed, the maximal incremental response will be reduced. As Naka and Rushton (1966) pointed out when they originally introduced the hyperbolic saturation equation, the new response curve has the same mathematical form 
as before, with a change in scales. If an original response to the incremental test was

$$
V_{t}=\frac{I_{i}}{I_{t}+\sigma}
$$

then the new one becomes

$$
V_{t}=\frac{\sigma}{\sigma^{\prime}}\left(\frac{I_{t}}{I_{t}+\sigma^{\prime}}\right)
$$

where

$$
\sigma^{\prime}=I_{0}+\sigma
$$

and

$$
I_{0}=\text { steady background signal. }
$$

A "subtractive" adaptation is a process that simply subtracts away part or all of the steady signal $I_{0}$, reducing it to a lower effective value. This is shown in Fig. 7(a). (This process belongs to the class of processes known as "additive", but the term "subtractive" will be used here to denote the fact that the sign of the added signal is negative.) The box marked " $A$ " generates a signal which is some fraction of the signal emerging from the linear stage, and this adapting signal is subtracted from the original signal at the summing junction. The adapting signal is zero initially, but over time it will grow and tend to subtract out the steadily present background response. Such an adaptation will have exactly the opposite effect that the steady background had; it will tend to lower the floor and expand the response range back to its original size, and in so doing cause $\sigma^{\prime}$, the effective semisaturation constant for increments, to return toward its original value. (Note that the choice of a feedforward scheme is arbitrary, as a similar model with subtractive feedback would have the same effects.)
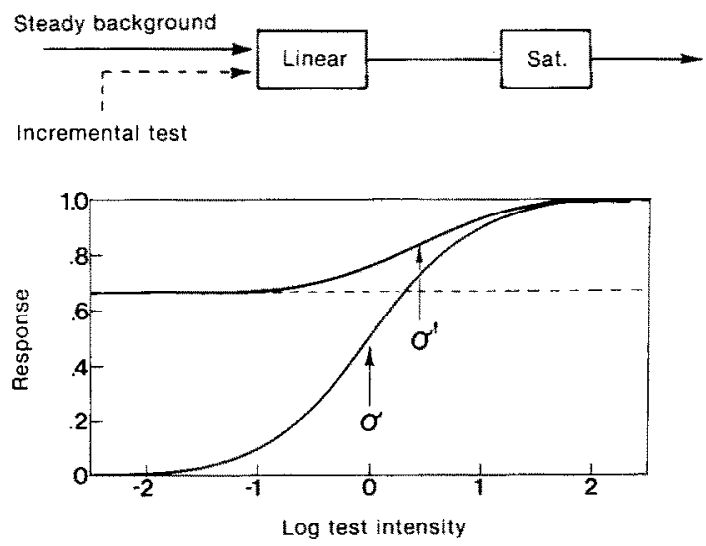

Fig. 6. Response compression caused by a steady signal added to a saturating stage of form $I /(I+\sigma)$. The new response function retains the same sigmoidal shape (when plotted on semi-log coordinates as here), with an increase in the effective value of $\sigma$, and a decrease in the available response range, $V_{\max }$.
The additive and subtractive effects will show up in the electrophysiology and the psychophysics as shown in Fig. 7(b) and (c). As backgrounds are added, in Fig. $7($ b), the response curves measured electrophysiologically will move down and to the right at $45^{\circ}$ on a $\log -\log$ plot. This is because both the maximal response size and the effective semisaturation constant are scaled by $\sigma /\left(I_{0}+\sigma\right)$. The effects of subtractive adaptation will be just the opposite: the curves will move up and to the left, toward their former position.

Psychophysically, the addition of a steady background signal raises the floor of the threshold curves, as shown in Fig. 7(c). The curves are of the form

$$
I_{t}=\frac{\delta\left(I+I_{0}+\sigma\right)^{2}}{k\left[\sigma-\delta\left(I+I_{0}+\sigma\right)\right]}
$$

where $I_{t}$ is the test intensity, $I$ is the background flash intensity, $I_{0}$ is the steady background signal, $k$ is the scale factor, $\sigma$ is the semisaturation constant, and $\delta$ is the criterion. In the case shown in the figure, $\delta=0.01$, $k=1$ and $\sigma=1$. The presence of $I_{0}$ has litle effect on the point of saturation until $I_{0}$ becomes quite large.

Once again, a subtractive adaptation will have the opposite effect, as it reduces the size of the steady signal entering the saturation stage. Thus, the floors of the psychophysical curves will move back downward, and the curves will fan out as shown.

Next, consider the case where the adaptive process is multiplicative, rather than subtractive. The model system is shown in Fig. 8(a), where the element labeled " $\mathrm{X}$ " represents the gain control, which is set by the adapting signal $G$.

A gain control by itself, with no additive effects from steady backgrounds, will result in the behavior shown in Fig. 8(b) and (c). The response curves (Fig. 8 b) will simply shift to the right by an amount equal to the log of the gain change. The increment threshold curves (Fig. 8c) will move up and to the right on a $45^{\circ}$ line. Because it occurs before the nun-linearity, the gain change acts just as a neutral density filter would.

In actual experiments, both additive and multiplicative processes may occur, and one may try to decompose the behavior into these two sets of effects.

\section{EXPERIMENT 4. TRANSIENT SATURATION WITH PRE-ADAPTATION}

To test how the adaptive state of the retina changed the increment threshold curves, a third field - a "preadapting" field-was used. This field was steadily present, and the background flash and test flash were presented against it. Two intensities of pre-adapting feld were used: $-0.8 \mathrm{log}$ scot. td, and $0.55 \mathrm{log}$ scot. td.

\section{Procedure}

The pre-adapting field was red (Wratten No. 26) and filled the same $11^{\circ}$ disc as did the flashed back- 


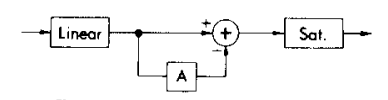

a.

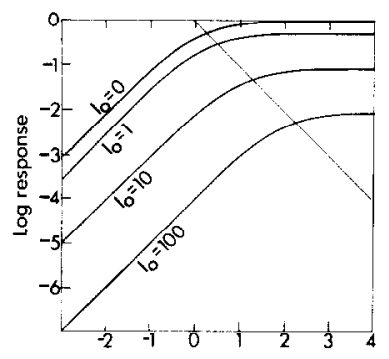

Log test intensity

b.

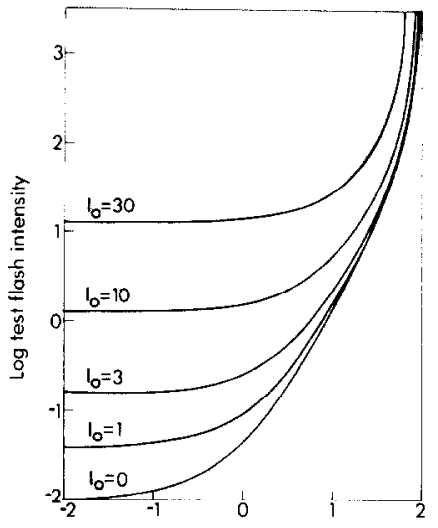

Log background flash intensity

Fig. 7. (a) Subtractive adaptation model. In response to a steady input. an adapting signal is generated by the box marked "A". and this signal is subtracted from the original signal at the summing junction marked " +". (b) The effect of a steady background signal, $I_{0}$, on the incremental response function of a system displaying $I /(I+\sigma)$ saturation, with no adaptation. Curves (on a log-log plot) shift down and to the right at $45^{\circ}$, due to response compression. A subtractive process can reverse the effect by reducing the size of $I_{0}$. (c) Changes in the corresponding increment threshold curves for the same situation, assuming $k=1, \sigma=1$ and $\delta=0.01$ (see text).

ground. The subject adapted to this field for at least two minutes before judging thresholds. Otherwise the stimulus was the same as in experiment 1: a $400 \mathrm{msec}$ red (Wratten No. 26) background was flashed, and a $480 \mathrm{~nm}$ test appeared for $30 \mathrm{msec}$, with simultaneous onset.

Results

As Fig. 9 shows, the increment threshold curves changed substantially under adaptation. The flashed background needed for saturation became much more intense as adaptation level increased. At the same time, the "absolute threshold"- the threshold for detecting a test in the absence of a flashed background - was also increased by the steady pre-adapting field's presence. Thus, the net result was a shifting of curves upward and to the right, as one would expect if a gain control were attenuating the signal before the saturating stage.

Note that a gain change alone cannot account for the data. The curves do not simply shift along a $45^{\circ}$ line; they also change shape somewhat. And the "absolute threshold" floors are shifted up to a greater extent than the saturating asymptotes are shifted to the right. Finally, the curve of equation (5) does not fit the shape of the dark adapted increment increment
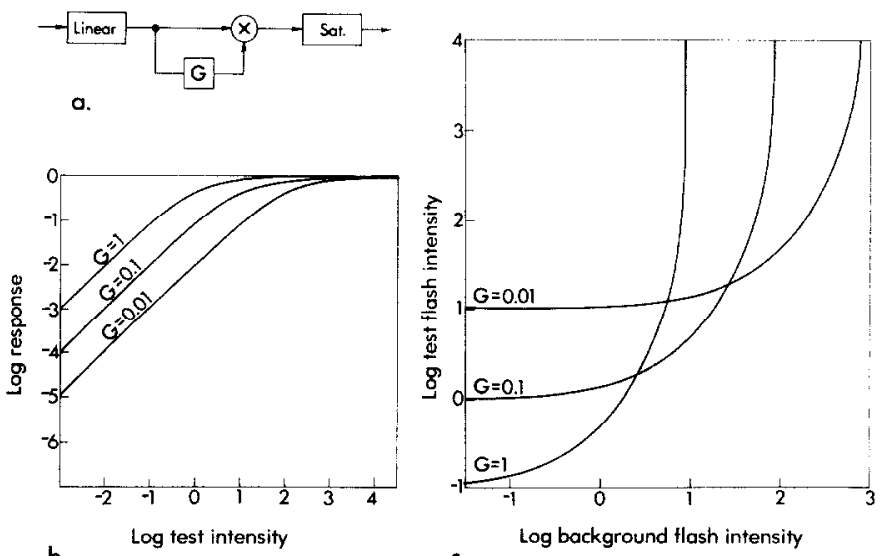

Fig. 8. (a) Multiplicative adaptation model. The adapting signal, generated at " $G$ ", multiplies the original signal by some gain between 0 and 1 , at the point marked " $X$ ". (b) The effect of a gain change on the response function of a system displaying $I /(I+\sigma)$ saturation. Curves shift to the right. (c) Changes in corresponding increment threshold functions for the same system. Curves shift up and to the right at $45^{\circ}$, without changing shape. 


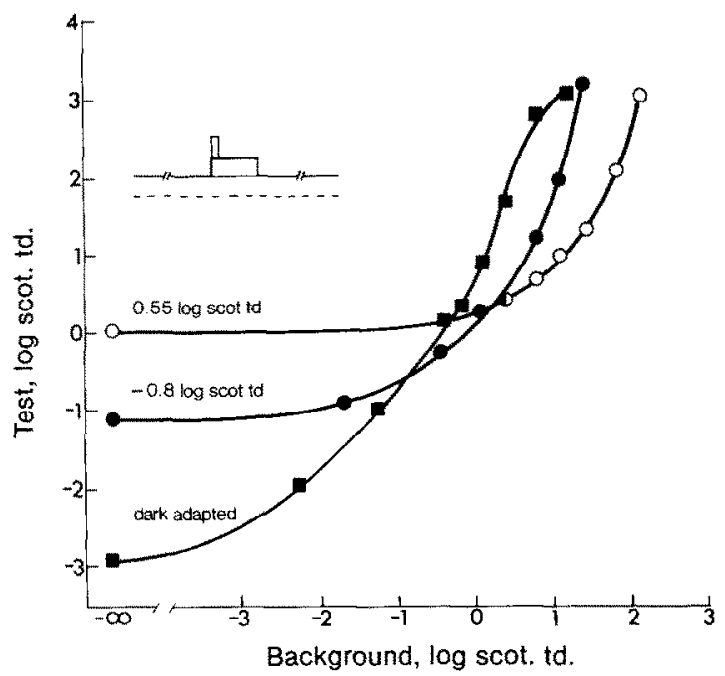

Fig. 9. Transient rod saturation, measured against steady preadapting fields. Pre-adapting field and flashed background were red (Wratten No. 26); $30 \mathrm{msec}$ test was blue $(480 \mathrm{~nm})$. Pre-adapting field intensities were $0.55 \mathrm{log}$ scot. td (open circles) or $-0.8 \mathrm{log}$ scot. td (solid circles) also shown is the case of no pre-adapting feld (solid squares). Subject E.A.

threshold curve terribly well; the model predicts that the data should rise more steeply than they actually do.

All of these defects might be remedied by allowing the saturating exponent, $n$, to be non-unity, and by choosing appropriate values of gain, and of subtractive adaptation, for each condition. But this would rapidly reduce to an exercise in curve-fitting that would go beyond the value of the data. The real significance of the data is this: a lincar system, followed by a hyperbolic saturation, can give data similar to those actually observed, if one assumes that steady adapting fields cause a reduction in gain prior to the saturating non-linearity. However, some response compression may be needed to explain the fact that the floor is raised more quickly than the saturating asymptote is shifted.

\section{EXPERIMENT 5. THE TIME COURSE OF ADAPTATION}

The previous experiment showed that a pre-adapting field can cause large changes in the threshold curves measured against flashed backgrounds. The next experiment was designed to elucidate the time course of the adaptive effect. The flashed background occurred at various times after the pre-adapting field had been turned on. Thus, the threshold was taken before the pre-adapting process had reached equilibrium.

\section{Procedure}

The red (Wratten No. 26) pre-adapting field appeared, and after some delay, the background flash and test flash appearcd simultancously. As before, the background was red (Wratten No. 26) and lasted $400 \mathrm{msec}$; and the test flash was blue $(480 \mathrm{~nm})$ and lasted $30 \mathrm{msec}$. The pre-adapting field always remained on for at least $400 \mathrm{msec}$ beyond the onset of the test.

\section{Results}

Figure 10 shows the increment threshold curves, one taken after a delay of $200 \mathrm{msec}$ (solid circles), and one taken against the same pre-adapting field, with "infinitc" dclay (i.c. stcady statc prcadaptation, shown in open circles, from Experiment 4). The pre-adapting field was $0.55 \log$ scot. td. Also shown, for comparison, are the thresholds obtained in the absence of pre-adaptation, from Experiment 1 (solid squares).

The pre-adapting field had a large and rapid effect on transient saturation. Within $200 \mathrm{msec}$, the point of saturation had shifted over to $2.2 \mathrm{log}$ scot. td-more than a log unit higher than it had been in the absence of a pre-adapting field.

Between $200 \mathrm{msec}$ and "infinity", the intensity of the background causing saturation changed hardly at all; the saturating asymptote did not shift to the right as one would expect from a gain change preceding saturation. However, the thresholds below saturation continued to fall slowly, until at equilibrium the "absolute threshold" against the pre-adapting field was $0.9 \log$ unit below its $200 \mathrm{msec}$ level; this is shown by the lowering of the floor of the threshold curve.

Figure 11 shows the time-course of the lowering of the floor in more detail. For this experiment, a $0.5 \mathrm{log}$ scot. td pre-adapting field was used, and no background flash was added. The test was superimposed on the adapting field at various times after its onset. Thus, these points describes the rate of fall of the threshold floors, such as would be seen in Fig. 10 against a $0.5 \log$ scot. td pre-adapting field. The two

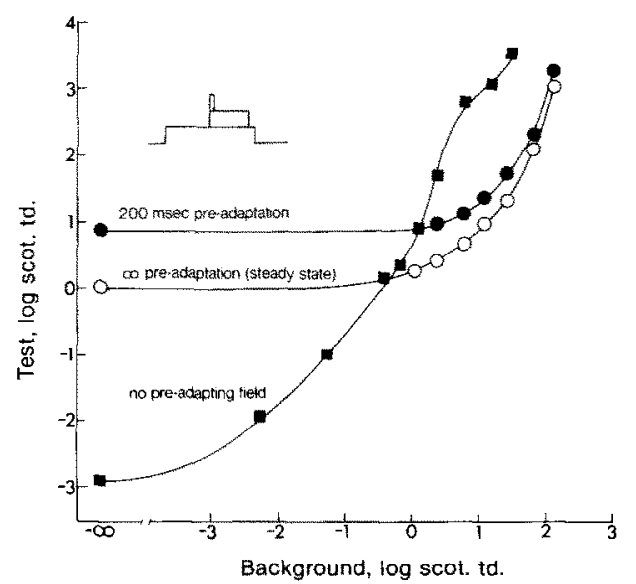

Fig. 10. Transient rod saturation, measured at $200 \mathrm{msec}$ after the onset of the pre-adapting field (solid circles), or after preadaptation had reached equilibrium (open circles). The pre-adapting field was $0.55 \mathrm{log}$ scot. td in both cases. Filled squares show the case of no pre-adapting field. Subject E.A. 


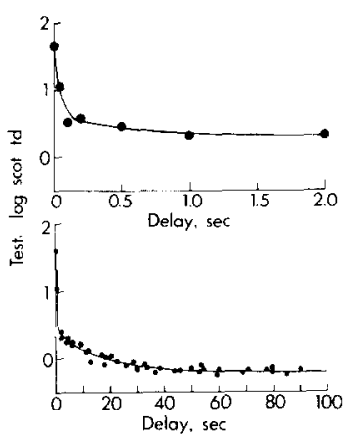

Fig. 11. Threshold for a $30 \mathrm{msec} 480 \mathrm{~nm}$ test flash, presented at various times after the onset of a $0.5 \mathrm{log}$ scot. td red (Wratten No. 26) field. Top and bottom show the same data, at different time scales.

parts of Fig. 11 show the results on two different time scales.

There seem to be two different kinds of adaptation in Figs. 10 and 11. One is very fast, and results in the rapid shift of the saturating asymptote within $200 \mathrm{msec}$. The other is much slower, and leads to the gradual lowering of the threshold floor over a period of about $1 \mathrm{~min}$.

There is some evidence for such fast gain changes in electrophysiology. Enroth-Cugell and Shapley (1973) have observed such an effect in recordings from cat retinal ganglion cells. The gain reduction asymptoted in less than $200 \mathrm{msec}$. Baylor and Hodgkin (1974) saw a very fast drop in gain in turtle cones, occurring within about $50 \mathrm{msec}$.

\section{DISCUSSION}

The data of Fig. 10 allow a confrontation between subtractive and multiplicative models of adaptation, as illustrated in Fig 12. In Experiment 5, the preadapting field of $0.55 \mathrm{log}$ scot. td was bright enough to cause transient saturation by itself at the instant of onset. This is suggested in Fig. 12 by the dashed line, which indicates that the response at $t=0$ is reaching saturation. Suppose now that the background flash,

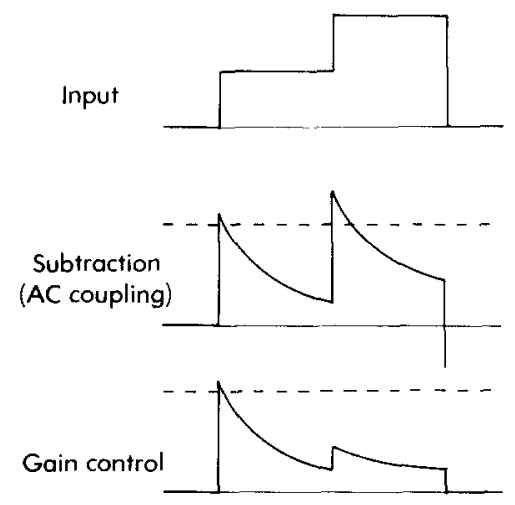

Fig. 12. Responses of subtractive (top) and multiplicative (middle) models to two step inputs of equal intensity (bottom). See text for details. added $200 \mathrm{msec}$ later, is of the same intensity. Then on the subtractive model (i.e. a.c.-coupling), the new flash response will be equal to the first one, and will be added to whatever response remains from the preadapting flash. Therefore, the new response will also be saturating. Conversely, on the gain control model (middle of Fig. 12), the new response will be attenuated by the reduced gain that the first flash induced. Thus, the second response will not reach the saturating ceiling.

The crucial difference between the two models is that under gain control, the tendency to saturation will be reduced by the preadapting flash, but under subtractive adaptation the saturation will be, if anything, slightly increased. The actual data show a reduction in saturation: within $200 \mathrm{msec}$, the background needed for saturation was considerably brighter than the one that had been needed in the absence of a pre-adapting field. If one must choose between these models, then, the gain control process certainly wins out, at least in the first $200 \mathrm{msec}$. (Of course, it remains possible that some subtraction occurs as well.)

Quite a different analysis applies to the slow changes that occur between $200 \mathrm{msec}$ and steady state pre-adaptation. Here, the lowering of the threshold floor, without the corresponding shift in the saturating asymptote, closely resembles the fanning out that should occur with a subtractive adaptation. In Fig. 13, two theoretical curves have been fit to the data of Fig. 10, to show that the transition from $t=0$ to $t=\infty$ is consistent with the subtractive model. The solid curves are members of the family generated by equation (9) and illustrated in Fig. 7. They differ only in the value of $I_{0}$, which is 13.1 at $200 \mathrm{msec}$ and 1.6 at $t=$ "infinity". The other parameters are: $\delta=0.03$, $\sigma=5$ and $k=0.284$. (Note that the parameter values can be traded off against one another for an equally good fit, so that these values are not unique.)

The fact that the two curves can be fit by varying a single paraneter $\left(I_{0}\right)$ points to an advantage of the present analysis over the " $V_{\max }, \sigma$ " approach more

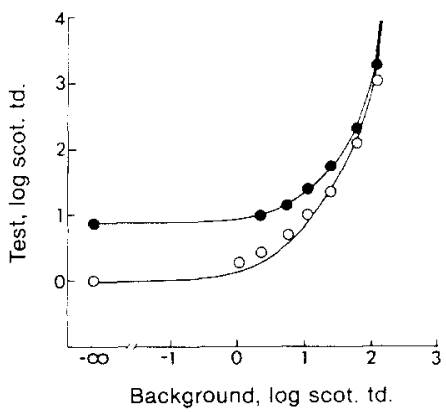

Fig. 13. The data of Fig. 10, on the time-course of preadaptation, fit with the subtractive model (equation 9). At $t=200 \mathrm{msec}, I_{0}=13.1$; at $t=\infty, I_{0}=1.6$. All other parameters are the same for both curves: $k=0.284, \sigma=5$ and $\delta=0.03$ 
commonly used. To accommodate the changes occurring in Fig. 13 via equation (1) (which is phrased in terms of $V_{\max }$ and $\sigma$ ) would require that both $V_{\max }$ and $\sigma$ be changed, and in the right proportions. But varying the single parameter $I_{0}$ in equation (9) transforms one curve into the other automatically.

One is led to a model containing both multiplicative and subtractive processes, each of which reduces the signal entering the saturating stage; one such scheme is shown in Fig. 14. The boxes marked "LP" represent low-pass filters, which generate the adaptive signals. The time constant associated with the gain control would be very fast; that associated with the subtraction would be much slower.

Geisler (1981) found evidence for a subtractive process in the human cone system, in experiments rather different from those discussed here. By briefly extinguishing a steady adapting field, he was able to separate out its additive effects (which vanished) and its multiplicative effects (which remained). Geisler proposed a model involving a fast subtractive process in the cone pathway, as contrasted with the slow subtractive process proposed in the present work on rods. Because the experiments are different, it is impossible to say whether homologous mechanisms are being studied. It may be that the same process is slow in rods and fast in cones, or it may be that the experiments reveal two different kinds of subtractive process, occurring at different points.

Two-process models, involving both multiplicative and additive components (recall that the process called "subtractive" constitutes an additive process), have also been used in studies of chromatic adaption (Hurvich and Jameson, 1958; Walraven, 1976; Shevell, 1978). In addition, recent work on the "blue" cone mechanisms, $\Pi_{1}$ and $\Pi_{3}$, has led Pugh and Mollon (1979) to develop a model incorporating both of these kinds of processes. It would be interesting to know whether the time constants of the additive and multiplicative effects are substantially different in these cases, as they seem to be for the rods.

The fast gain change proposed here also provides an explanation for the curious dark appearance of the test patch mentioned in Experiment 1. When transient saturation occurs, the responses to both the test and background regions are saturated, and are therefore indistinguishable. However, the test region, being more intense than the background, will turn down the gain more than the background region will. Then, the $400 \mathrm{msec}$ background flash, which remains on after

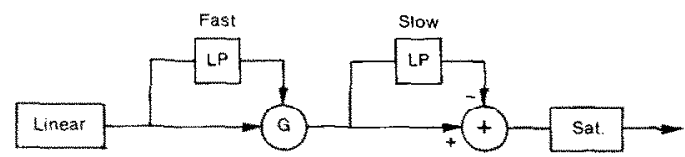

Fig. 14. The two-process model for adaptation in the rod system. The multiplicative process is controlled by a lowpass filter (L.P.) of short time-constant, and exerts its effect at $G$. The subtractive process, which exerts its influence at " +", has a much longer time constant. the test flash, will be multiplied by a lower gain in the test region than it will be in the surrounding region, and therefore the test region will appear darker. (A similar process may underly "Bidwell colors", in which a negative afterimage is induced without a positive image having been visible $\mathrm{cf}$. Sperling, 1960.)

Following this idea, I have made powerful negative afterimages, by the following method: in the peripheral retina, I present a $50 \mathrm{msec}$ flash of blue $(480 \mathrm{~nm})$ light at $1 \log \mathrm{scot}$. $t$, and $200 \mathrm{msec}$ later present a large uniform flash of the same color and intensity. The bright appearance of the test immediately reverses to dark when the second flash appears, apparently because the lower gain multiplies the uniform field.

In the original experiments, the choice of criterion can have a large effect on the data, as illustrated in Fig. 15. The procedure here was the same as the flashed condition of Experiment 1: no preadaptation, and a $30 \mathrm{msec}$ test against a $400 \mathrm{msec}$ background with simultaneous onset. However, in the present case the subject used two different criteria for threshold. The first was to see the test as distinctly lighter than the background; the second was to detect the test in any way possible (e.g. as a lighter or darker patch). For flashed backgrounds above $0 \mathrm{log}$ scot. td, it is much easier to see the dark-appearing image of the test than the light one. Thus, saturation measured by the "detect by any means" criterion occurs at brighter backgrounds than it does when the criterion is to see the test as lighter than the background.

These effects present a dilemma for the psychophysicist studying transient saturation. In a forcedchoice experiment the preferred paradigm, in many cases detection via the fast negative afterimage will be confounded with detection by the usual positive

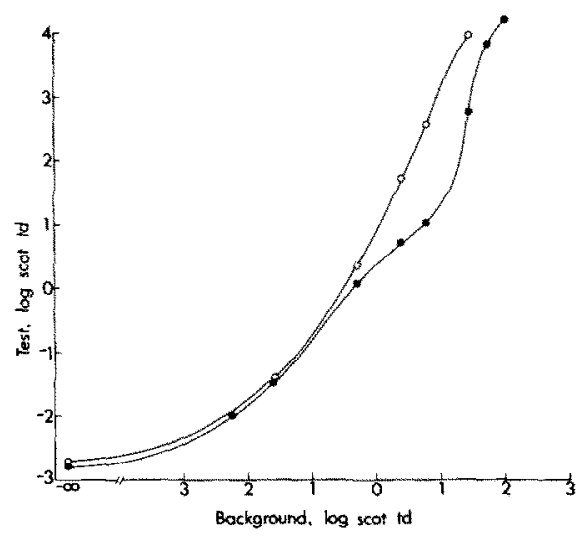

Fig. 15. Threshold for a $30 \mathrm{msec} 480 \mathrm{~nm}$ test flash, presented against a $400 \mathrm{msec}$ red (Wratten No. 26) background with simultaneous onset. Two criteria were used for detection. Open circles: the test appeared distinctly lighter than the background. Solid circles: the test could be distinguished by any means. Above olog scot. td, the threshold for the test to appear dark is substantially lower than the threshold for it to appear light. Subject A.B. 
response. One has two options: one can abandon forced-choice, and require that the subject adopt some criterion beyond "detection by any means"; or one can allow detection by any means, and afterwards try to disentangle the various effects that are mixed in the data. I have adopted the former strategy, in hopes of making the analysis simpler.

One other model should be mentioned. To deal with afterflash experiments, Alpern et al. (1970a) proposed an adaptational gain control that occurred after the saturating stage, rather than before it. The prediction, for Fig. 9, would be that the saturating asymptote should move leftward as the floor is raised; the actual data are not in accord with this prediction.

\section{Correlation with the electrophysiology}

This discussion has invoked many concepts from electrophysiology, without specifying the exact physiological mechanisms that might be involved. At present, it is impossible to say precisely where the saturation occurs, and where the adaptive processes take place. Some of the effects may involve the receptors themselves, but probably not all.

The transient saturation appears at about $0.5 \mathrm{log}$ scot. td in the dark adapted eye. If we suppose that only the first $200 \mathrm{msec}$ of the background flash are effective in causing the saturation, this translates to $0.63 \log$ td-sec, or about 3.8 quanta absorbed per rod, using Westheimer's (1966) conversion, corrected by Alpern and Pugh's (1974) more recent estimate of human rhodopsin density. This is some 10 times lower than the 30-40 quanta per rod found to cause semisaturation in rat rods (Penn and Hagins, 1972), and it seems quite unlikely that such a small quantum catch could cause saturation at the receptor level. This would suggest that the saturation occurs beyond the receptors themselves, perhaps at the bipolar or ganglion cells. Alexander's (1980) results on rod-cone interactions lend further evidence for such post-receptoral processes.

The transient saturation effect is greatly reduced within a few hundred milliseconds. When the test is delayed by $1000 \mathrm{msec}$, as in Fig. 4, saturation begins around $1.9 \log$ scot. td, which translates to about 470 quanta absorbed per rod per second. And this is very close to the $350 \mathrm{q} \mathrm{rod}^{-1} \mathrm{sec}^{-1}$ that Penn and Hagins found to give half-saturation in rat rods with a $2 \mathrm{sec}$ flash of light.

It is conceivable, then, that the initial saturation represents a stage beyond the receptors. However, a post-receptoral adaptation could very quickly attenuate the signal sufficiently to avoid this saturation, and brighter lights would then run into the saturating ceiling imposed by the receptors themselves. If one supposes that the saturation observed at $1000 \mathrm{msec}$ is in the rods, then one must propose that human rods adapt, since in steady adaptation conditions rod saturation appears with backgrounds that are substantially brighter than those which saturate at $1 \mathrm{sec}$. It is sometimes said that mammalian rods do not adapt (Penn and Hagins, 1972; Green, 1973; Dowling, 1967), but this may be open to question, as is discussed in appendix II.

Adaptation certainly does occur in the rods of some cold-blooded animals, and behaves in ways consistent with the results of the present experiments. For example, Fain (1976), measured the response-intensity functions for rods of Bufo marinus, at various times after turning on a bright adapting field. At $24 \mathrm{sec}$ (the earliest measurements made) the curves were shifted several log units to the right, indicating a large reduction in gain, and slightly down, suggesting some compression as well. Over a period of $4 \mathrm{~min}$, the response functions shifted up and to the left along a line of approximately $45 \mathrm{deg}$, as would be predicted from a slow subtractive adaptation. Similar effects have been observed by Hemilä (1977) in frog rods. He has interpreted them of a fully elaborated physiological model, which has some similarities to the simple functional model used here.

Thus, it remains possible that many of the effects that have been measured psychophysically in the present experiments reflect processes at the receptor level; however, they may also result from post-receptoral processes that are functionally equivalent.

Acknowledgements-w-This work was performed in partial fulfillment of the requirements for the degree of doctor of philosophy at the University of Michigan. The author was supported by National Institute of Mental Health Training grant T32-MH14254 and by National Eye Institute grant EY00197 to M. Alpern. Dr Alpern's contribution of knowledge, advice, and laboratory facilities are gratefully acknowledged. The work has also benefitted greatly from discussions with Dr Angela Brown, Dr Daniel Green, Dr Donald Hood, Dr Wilson Geisler and Dr David Krantz.

\section{REFERENCES}

Adelson F. H. (1977) Transient rod saturation with moderate stepped backgrounds. A. R. V. O. Sarasota, FL.

Aguilar M. and Stiles W. S. (1954) Saturation of the rod mechanism of the retina at high levels of illumination. Optica Acta 1, 59-65.

Alexander K. R. (1980) Cone activity from flashed backgrounds impairs rod vision (abstr.). J. opt. Soc. Am. $\mathbf{7 0 ,}$ 1615.

Alpern M. and Pugh E. N. Jr (1974) The density and photosensitivity of human rhodopsin in the living retina. $J$. Physiol. 237, 341-370.

Alpern M., Rushton W. A. H. and Torii S. (1970b) The attenuation of rod signals by backgrounds. J. Physiol. 206, 209-227.

Alpern M.. Rushton W. A. H. and Torii S. (1970b) Signals from cones. J. Physiol. 207, 463-475.

Barlow H. B. (1958) Temporal and spatial summation in human vision at different background intensities. $J$. Physiol. 141, 337-350.

Baylor D. A. and Fourtes M. G. F. (1970) Electrical responses of single cones in the retina of the turtle. $J$. Physiol. 207, 77-92.

Baylor D. A. and Hodgkin A. L. (1974) Changes in time scale and sensitivity in turtle photoreceptors. J. Physiol. 242, 729-758.

Boynton R. M. and Kandel G. (1957) On responses in the human visual system as a function of adaptation level. $J$. opt. Soc. Am. 47, 275-286. 
Boynton R. M. and Whitten D. N. (1970) Visual adaptation in monkey cones: recordings of late receptor potentials. Science 170, 1423-1426.

Brindley G. S. (1959) The discrimination of afterimages. $J$. Physiol. 147, 194-203.

Brindley G. S. (1963) Afterimages. Scient. Am. 209, Oct. 84-91.

Crawford B. H. (1947) Visual adaptation in relation to brief conditioning stimuli. Proc. R. Soc. B 134, 283-302.

Dowling J. E. (1967) Site of visual adaptation. Science 155, 273-279.

Dowling J. E. and Ripps H. (1972) Adaptation in skate photoreceptors. J. gen. Physiol. 60, 698-719.

Enroth-Cugell C. and Shapley R. M. (1973) Adaptation and dynamics of cat retinal ganglion cells. $J$. Physiol. 233, 271-309.

Fain G. L. (1976) Sensitivity of toad rods: dependence on wavelength and background illumination. $J$. Physiol. 261, 71-101.

Flamant $\mathbf{F}$. and Stiles W. S. (1948) The directional and spectral sensitivities of the retinal rods to adapting fields of different wavelengths. $J$. Physiol. 107, 187-202.

Gcisler W. S. (1978) Adaptation, afterimages and cone saturation. Vision Res. 18, 279-289.

Geisler W. S. (1979) Initial image and afterimage discrimination in the human rod and cone systems. $J$. Physiol. 294, $165-179$.

Geisler W. S. (1980) Increment threshold and detection latency in the rod and cone systems. Vision Res. 20, 981-994.

Geisler W. S. (1981) Effects of bleaching and backgrounds on the flash response of the cone system. J. Physiol. 312, 413-434.

Green D. G. (1973) Scotopic and photopic components of the rat electroretinogram. J. Physiol. 228, 781-797.

Hallett P. E. (1969) Rod increment thresholds on steady and flashed backgrounds. $J$. Physiol. 202, 355-377.

Hemilä S. (1977) Background adaptation in the rods of the frog's retina. J. Physiol. 265, 721-741.

Hood D. C. (1978) Psychophysical and physiological tests of proposed physiological mechanisms of light adaptation. In Visual Psychophysics: Its Physiological Basis (Edited by Armington J., Krauskopl J. and Wooten B.). Academic Press, New York.

Hood D. C., Finkelstein M. A. and Buckingham E. (1979) Psychophysical tests of models of the response function. Vision Res. 19, 401-406.

Hood D. C., Ilves T., Maurer E., Wandell B. and Buckingham E. (1978) Human cone saturation as a function of ambient intensity: a test of models of shifts in the dynamic range. $V$ ision Res. 18, 983-993.

Hurvich L. M. and Jameson D. (1958) Further developments of a quantified opponent-colors theory. In Visual Problems of Colour II, pp. 691-723. H.M.S.O., London.

King-Smith P. E. and Webb J. R. (1974) The use of photopic saturation in determining the fundamental spectral sensitivity curves. Vision Res. 14, 421-429.

Kleinschmidt J. and Dowling J. E. (1975) Intracellular recordings from gecko photoreceptors during light and dark adaptation. J. gen. Physiol. 66, 617-648.

Naka K. I. and Rushton W. A. H. (1966) S-potentials from luminosity units in the retina of fish (Cyprinidae). J. Phy. siol. 185, 587-599.

Norman R. A. and Werblin F. S. (1974) Control of retinal sensitivity. 1. Light and dark adaptation of vertebrate rods and cones. J. gen. Physiol. 63, 37-61.

Penn R. D. and Hagins W. A. (1972) Kinetics of the photocurrent of retinal rods. Biophys, J. 12, 1073-1094.

Pugh E. N. and Mollon J. D. (1979) A theory of the $\Pi_{\mathrm{t}}$ and $\Pi_{3}$ color mechanisms of Stiles. Vision Res. 19. 293-312.

Shevell S. K. (1977) Saturation in human cones. Vision Res. $17,427-434$
Shevell S. K. (1978) The dual role of chromatic backgrounds in color perception, Vision Res. 18, 1649-1661.

Sperling G. (1960) Negative afterimage without prior positive image. Science 131, 1613-1614.

Sperling G. (1965) Temporal and spatial visual masking. I. Masking by impulse flashes. $J$. opt. Soc. Am. 55, 541-559.

Tomita T. (1968) Electrical responses of single photoreceptors. Proc. I.E.E.E. 56, $1015-1023$.

Walraven J. (1976) Discounting the background the missing link in the explanation of chromatic induction. Vision Res. 16, 289-295.

Werblin F. S, and Dowling J. E. (1969) Organization of the retina of the mudpuppy, Necturus maculosus, II. Intracellular recording. $J$. Neturophysiol. 32, 339-355.

Westheimer G. (1966) The Maxwellian view. Vision Res. 6, 669-682.

White T. W., Kelly S. A. and Sturr J. F. (1978) Large field early light adaptation. Vision Res. 18, 1679-1684.

Williams T. P. and Gale J. G. (1977) A critique of an incremental threshold lunction. Vision Res. 17, 881-882.

Wyszecki G. and Stiles W. S. (1967) Color Science. Wiley, New York.

\section{APPENDIX I}

\section{A.C. coupling as a subtractive process}

A resistor and a capacitor, joined as shown in Fig. 16(a), form a single stage high-pass filter. This arrangement is used, for example, on the input stage of oscilloscopes on the "a.c." setting.

The response of the system to a unit step is

$$
V_{\text {out }}=\exp (-t / \tau)
$$

where $\tau=\mathrm{RC}$, the time constant.

Another way of achieving the same response is shown below, in Fig. 16(b). Here, the output of a low-pass filter, whose step response is $1-\exp (-t / \tau)$, is subtracted from the input signal via feed-forward. As illustrated in the lower right, the arithmetic difference is $1-[1-\exp (-t /$ $\tau)]=\exp (-t / \tau)$, so that the net step response is identical to that of the RC network shown above.

Since both systems are linear, they are completely characterized by their step responses; and since their step responses are identical, they will behave identically for all inputs.

\section{APPENDIX II}

\section{A second look at Penn and Hagins}

Penn and Hagins (1972), in their seminal paper on photocurrent in rat rod, found that a rod could be modelled as a linear system followed by a saturation of form $I /(I+\sigma)$. They claimed that this model could explain the responses to long and short pulses, without invoking

(a)

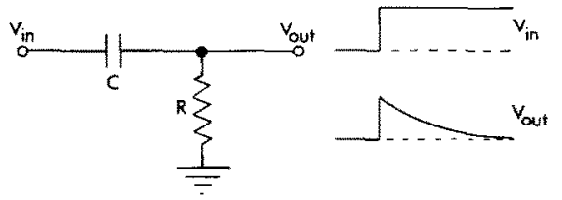

(b)

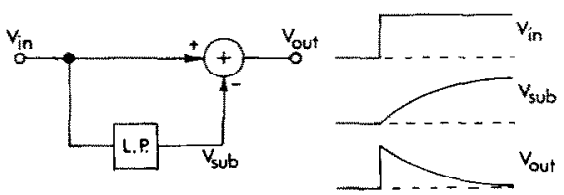

Fig. 16. (a) Simple "RC" high-pass filter, along with its step response (right). (b) The equivalent system constructed with a lowpass filter and feed-forward subtraction. 


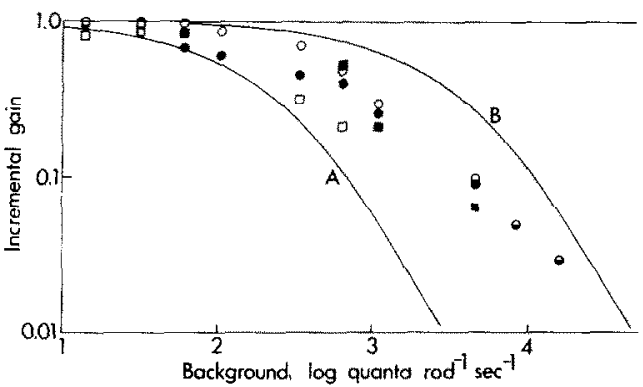

Fig. 17. Re-analysis of the data of Penn and Hagins (1972). Data points represent incremental gain of rat rods, for brief flashes superimposed on $2 \mathrm{sec}$ backgrounds of various intensities. Curve " $A$ " is the result predicted from Penn and Hagins' model, optimized for dark adapted case. Curve " $B$ " is the prediction from the same model optimized to fit human rod saturation data.

any adaptation within the rod, and that it could quantitatively explain the saturation observed psychophysically by Aguilar and Stiles (1954).

Penn and Hagins did not explicitly state the assumptions under which they obtained this match between model, physiology and psychophysics; however, using what would seem to be the most reasonable assumptions leads to serious discrepancies. The original model, applied in a straightforward way, does not fit the physiological data on adaptation, nor does it quantitatively match the saturation of Agular and Stiles.

Figure 17 shows the data Penn and Hagins obtained on the incremental gain of rod photocurrent, when brief increments were superimposed on $2 \mathrm{sec}$ adapting fields of various intensities. The incremental flash was of fixed intensity, and the gain was taken to be proportional to the response that it elicited.

The curve on the left, labelled "A", is the function predicted from Penn and Hagins' model, using a string of four low-pass stages with time constants of $35.2,35.2,89.3$ and $89.3 \mathrm{msec}$, which were the parameters those authors found to give the best fit for the responses to brief flashes. The curve was generated by simulating the model's response on a computer. The change in incremental gain is due entirely to the saturation, and the curve takes the same form as equation (9), for small $\delta$, except that it is inverted due to the direction of the ordinate. The semisaturation constant was chosen to give a half-maximal response to a brief flash of $1.6 \mathrm{log}$ quanta per rod (again from the measurements of Penn and Hagins); this gave a steady half-maximal response with an input of $2.1 \log$ quanta $\operatorname{rod}^{-1} \mathrm{sec}^{-1}$, using the dynamics of the model.

At low and moderate light levels, sensitivity rematins constant, as a non-adapting model would require. But at higher light levels, the data deviate from the prediction. Not only is curve A too far to the left of the actual data. but it falls too steeply. The model predicts that the fall-off in gain will asymptotically approach a slope of -2 whereas the actual data seem to show a slope of -1 . (Penn and Hagins actually show a "predicted" curve in which the slope does approach -1 , but this seems to be an error.) The shallow slope displayed in the data is consistent with an adapting mechanism that counteracts the tendency to saturation at high light levels.

To compare the physiological model with Aguilar and Stiles data, one needs a way of estimating the semisaturation constant from an increment threshold curve. It is not obvious how to do this, and Penn and Hagins do not state how they chose to do it. A reasonable approach would be to assume that Weber's law holds in the absence of saturaion, and to take deviations from the Weber line as indicating losses in incremental gain. On this assumption. halfsaturation in human rods should occur at about $2.5 \mathrm{log}$ scot. $t$, or $3.3 \log$ quanta rod ${ }^{-1}$ sec $^{-1}$ (converted according to Westheimer, 1966, corrected by Alpern and Pugh. 1974). The corresponding curve is shown in Fig. 17 as curve " $B$ ": it is well to the right of the data.

Penn and Hagins' own data on adaptation fall in between. Some order may be found here: the model based on brief flashes (curve A) exhibits saturation at low intensities; the data points shown, which involved modest amounts of adaptation ( $2 \mathrm{sec}$ ) show saturation at an intermediate level; and the psychophysics (curve B), which involved prolonged adaptation, shows the highest intensity for saturation. This point should not be pushed too hard. since the comparisons between specics and cxperiments involve many assumptions. But the story on rod saturation and adaptation seems incomplete. 